\title{
Basic fibroblastic growth factor affects the osteogenic differentiation of dental pulp stem cells in a treatment-dependent manner
}

\author{
J. Qian ${ }^{1}$, W. Jiayuan ${ }^{2}$, J. Wenkai ${ }^{1}$, W. Peina ${ }^{1}$, Z. Ansheng ${ }^{1}$, S. Shukai ${ }^{1}$, Z. Shafei ${ }^{1}$, \\ L. $\operatorname{Jun}^{3}$ \& N. Longxing ${ }^{1}$ \\ ${ }^{1}$ State Key Laboratory of Military Stomatology, Department of Operative Dentistry \& Endodontics, School of Stomatology, \\ Fourth Military Medical University, Xi'an, Shaanxi; ${ }^{2}$ Hospital of Stomatology, The Zunyi Medical University, Zunyi, Guizhou, \\ China; and ${ }^{3}$ Department of Cariology, Restorative Sciences and Endodontics, University of Michigan, Ann Arbor, MI, USA
}

\begin{abstract}
Qian J, Jiayuan W, Wenkai J, Peina W, Ansheng Z, Shukai S, Shafei Z, Jun L, Longxing N. Basic fibroblastic growth factor affects the osteogenic differentiation of dental pulp stem cells in a treatment-dependent manner. International Endodontic Journal, 48, 690-700, 2015.
\end{abstract}

Aim To determine how basic fibroblastic growth factor (bFGF) affected the osteogenic differentiation of human dental pulp stem cells (DPSCs) in vitro and in vivo.

Methodology Basic fibroblastic growth factor stimulation of DPSCs was divided into a pre-treatment period and an osteogenic differentiation period. Alizarin red quantification experiments and alkaline phosphatase activity quantification assay were performed to examine the osteogenic differentiation of DPSCs after different bFGF stimulation. Quantification reverse transcription polymerase chain reaction was used to analyze the osteogenic gene expression of DPSCs after different bFGF stimulation. In addition, DPSCs that received the 1 and 2 weeks bFGF pre-treatments as in the in vitro experiments were mineralized for 1 week and seeded into hydroxyapatite/tricalcium phosphate (HA/TCP) pills and subcutaneously transplanted into naked mice for 2 or 3 months. The transplants were removed, sliced and stained using Modified Ponceau Trichrome Stain to observe the formation of mineralized tissue.

Results Basic fibroblastic growth factor stimulation in the osteogenic differentiation period decreased the in vitro osteogenic differentiation ability of DPSCs. One week pre-treatment with bFGF increased the in vitro osteogenic differentiation ability of DPSCs, whereas 2 weeks pre-treatment with bFGF decreased the in vitro osteogenic differentiation ability of DPSCs. The pre-treatment period was vital for the osteogenic differentiation of DPSCs in vitro. The in vivo results were similar to the in vitro results.

Conclusions Basic fibroblastic growth factor affected the osteogenic differentiation of DPSCs in a treatment-dependent manner both in vitro and in vivo.

Keywords: basic fibroblast growth factor, dental pulp stem cells, osteogenic differentiation.

Received 15 January 2014; accepted 6 August 2014
Correspondence: Ni Longxing and Liu Jun, State Key Laboratory of Military Stomatology, Department of Operative Dentistry \& Endodontics, School of Stomatology, Fourth Military Medical University, No.145 Western Changle Road, Xi'an, Shaanxi 710032, China (Tel.: +86 29 84776476; Fax: +86 29 83224432; e-mails: nilx2007@gmail.com, junlc@umich. edu).

Qian J and Jiayuan W contributed equally to this work.

\section{Introduction}

Oral stem cells include dental pulp stem cells (DPSCs), periodontal ligament stem cells and stem cells from the apical papilla, Gronthos et al. (2000) first reported that stem cells isolated from adult dental pulp could be cultured in vitro. In addition, this type of cell could differentiate into odontoblasts and could form dentine-like tissue. Since then, the concept of DPSCs has been proposed. DPSCs have been characterized as cells 
that are capable of self-regeneration, have a high degree of proliferation and have the potential to differentiate into multiple types of cells.

Basic fibroblastic growth factor (bFGF) is a mitogenic cationic peptide that consists of 155 amino acids that is extensively distributed throughout the human body. As a cell mitogen, it mainly impacts on mesoblasts and neuroderm-oriented cells, such as skeletal muscle cells, fibroblasts and osteocytes. Correspondingly, its receptor is expressed on these cells. In addition to its mitogenic effects, bFGF is a morphogenesis and differentiation inducer. The main biological effects of bFGF include (i) its role as an angiogenic factor, (ii) its promotion of wound healing and tissue restoration, (iii) its promotion of tissue regeneration, and (iv) its participation in neurogenesis (Davidson et al. 1985, Lobb et al. 1985, Abraham et al. 1986, Cuny et al. 1986, Canalis et al. 1987).

Many in vivo studies have shown that bFGF can induce bone regeneration (Mayahara et al. 1993, Nakamura et al. 1995, Zellin \& Linde 2000). However, the in vitro effects of bFGF on cell osteogenic differentiation remain unclear. Some studies have shown that stimulation with bFGF inhibited the production of collagen and alkaline phosphatase (ALP) and the osteogenic differentiation of osteoblasts (Canalis et al. 1988, Varghese et al. 1995, Tang et al. 1996). In contrast, other reports have shown that bFGF enhanced the osteogenic differentiation of multipotent stem cells (MSCs), and osteoblasts if the cells were exposed to bFGF only during the late periods of cell osteogenic differentiation (Debiais et al. 1998, Tsutsumi et al. 2001, Fakhry et al. 2005). In addition, it has been demonstrated that bFGF could induce the dexamethasone-dependent osteogenic differentiation of MSCs and that combined treatment with bFGF and BMP-2 synergistically enhanced the osteogenic potency of bFGF in MSC cultures (Hanada et al. 1997).

Several years ago, researchers began to focus on the relationship between bFGF and human dental pulp cells (HDPCs)/DPSCs (He et al. 2008, Kim et al. 2010, Osathanon et al. 2011). However, similar disagreement was found regarding the in vitro effects of bFGF on HDPCs/DPSCs. Some articles have reported that bFGF increased ALP activity and enhanced osteogenic differentiation in the HDPCs/DPSCs (He et al. 2008, Kim et al. 2010). However, other reports showed that bFGF inhibited the osteogenic differentiation of HDPCs/DPSCs (Shimabukuro et al. 2009, Osathanon et al. 2011). A review of these articles on
bFGF and HDPCs/DPSCs revealed that the stature of the cells and the treatment protocol of bFGF were different. (Shiba et al. 1995, He et al. 2008, Shimabukuro et al. 2009, Kim et al. 2010, Osathanon et al. 2011). Therefore, an experiment was designed that was as comprehensive as possible to determine the relationship between bFGF and the osteogenic differentiation of DPSCs in vitro and in vivo.

Hydroxyapatite/tricalcium phosphate (HA/TCP) has been commonly used as a scaffold material for in vivo experiments in stem cell research. Its biological characteristics make it suitable for use in stem cell research, especially for in vivo osteogenic differentiation assays (Zhang et al. 2006). Shi et al. (2005) used this material to observe the osteogenic characteristics of MSCs (mesenchymal stem cells). Therefore, HA/ TCP was used in the classic manner, which involves seeding DPSCs into HA/TCP and inserting the complex into the backs of naked mice to observe how the in vivo osteogenic differentiation of the DPSCs was affected by bFGF stimulation, according to the treatment method.

\section{Materials and methods}

\section{Sample collection and cell culture}

Human impacted third molars were collected from adults (19-29 years of age) at the dental hospital of the Fourth Military Medical University. The dental pulp was isolated as previously described (Gronthos et al. 2000). All the tooth extractions were conducted under the approval of the Ethical Committee of the hospital. Briefly, the pulp tissue was gently separated from the crown and root and then was digested in a solution of $3 \mathrm{mg} \mathrm{mL}^{-1}$ collagenase type I (Invitrogen, Carlsbad, CA, USA) and $4 \mathrm{mg} \mathrm{mL}^{-1}$ dispase (GIBCO/ Invitrogen, Carlsbad, CA, USA) for $40 \mathrm{~min}$ at $37^{\circ} \mathrm{C}$. Single-cell suspensions of DPSCs were obtained by passing the solution through a $70-\mu \mathrm{m}$ strainer and seeding $1 \times 10^{4}$ cells well $^{-1}$ onto six-well plate (Corning Costar, Cambridge, MA, USA). The standard medium was defined as: $\alpha$-modification of Eagle's Medium ( $\alpha$-MEM; GIBCO/Invitrogen) supplemented with $10 \%$ foetal bovine serum (FBS; HyClone, Kerrville, TX, USA); $100 \mathrm{~mol} \mathrm{~L}^{-1}$ L-ascorbic acid 2-phosphate (Sigma, St Louis, MO, USA); $2 \mathrm{mmol} \mathrm{L}^{-1} \mathrm{~L}^{-}$ glutamine (Sigma); $100 \mathrm{U} \mathrm{mL}^{-1}$ penicillin; and $100 \mu \mathrm{g} \mathrm{mL}^{-1}$ streptomycin (GIBCO) at $37^{\circ} \mathrm{C}$ in $5 \%$ $\mathrm{CO}_{2}$. The medium was changed every 2-3 days. Single cells were isolated using the limiting dilution 
technique, and they were seeded onto a 96-well plate and were allowed to grow into a single colony. A number of these single-colony-derived strains of DPSCs were pooled, cultured and passaged at a $1: 3$ ratio when they reached $80 \%$ confluence. Next, the cells were stained with STRO-1, CD146, vimentin (Sigma), and keratin (Sigma) using immunofluorescence staining. Laser scanning confocal microscopy showed that the cells were STRO-1, CD146 and vimentin positive and keratin (negative control) negative. The experiments were performed using the cells from the third to sixth passages. bFGF (Calbiochem, San Diego, CA, USA) was prepared according to the manufacturer's instructions and was diluted to a concentration of $10 \mathrm{ng} \mathrm{mL} \mathrm{m}^{-1}$ in standard medium ( $\alpha$-MEM containing $10 \%$ FBS) or standard osteogenic differentiation medium [standard medium containing $50 \mathrm{mg} \mathrm{mL}^{-1}$ ascorbic acid (Sigma), $10 \mathrm{mmol} \mathrm{L}^{-1}$ beta-glycerophosphate (BGP; Sigma) and $10 \mathrm{ng} \mathrm{mL}^{-1}$ dexamethasone], as previously described (Osathanon et al. 2011).

\section{In vitro osteogenic differentiation assay}

The in vitro osteogenic differentiation assay was divided into five small assays to detect the effects of bFGF on the osteogenic differentiation of DPSCs. In assays 1, 2, 3 and 4, DPSCs were seeded into a 24-well plate at a density of approximately $1 \times 10^{5}$ cells per well and were divided into experimental and control groups. After reaching 80\% confluence, the cells in the control and experimental groups were treated with or without bFGF according to Table 1 . In assay 5, DPSCs were seeded into two $25-\mathrm{cm}^{2}$ cell culture flasks (Corning Incorporated, Corning, NY, USA) at a density of approximately $1 \times 10^{6}$ and were divided into experimental and control groups. After reaching $80 \%$ confluence, the cells in the experimental group were pre-cultured in stan-

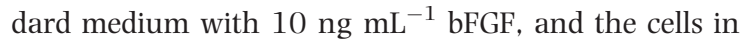
the control group were pre-cultured with standard medium for 1 week. Then, the cells were seeded into a 24-well plate at a density of approximately $1 \times 10^{5}$ cells per well after subcultivation. The experimental and control groups were cultured with standard osteogenic differentiation medium for 4 weeks according to Table 1 . After cultivation, all of the cells were stained with Alizarin red for Alizarin red quantification experiments, according to the methods developed by Osathanon et al. (2011). All of the experiments were performed at least three times.

\section{ALP activity quantification}

The DPSCs (200 cells well ${ }^{-1}$ ) were seeded onto a 96well plate and were divided into three groups. Each group had five wells. In this assay and the following assays, the cells pre-treated with standard media with bFGF (10 $\mathrm{ng} \mathrm{mL}^{-1}$ ) for 1 week were designated as group 1, the cells pre-treated with standard media

Table 1 In vitro osteogenic differentiation assay

\begin{tabular}{|c|c|c|c|c|}
\hline Assay & Group & Cell condition & Pre-treatment & Osteogenic differentiation for 4 weeks \\
\hline \multirow[t]{2}{*}{ Assay 1} & EXP & Confluence & $-1-$ & $\begin{array}{l}\text { Osteogenic differentiation medium } \\
\text { with bFGF }\end{array}$ \\
\hline & CTR & Confluence & $-1-$ & Osteogenic differentiation medium \\
\hline \multirow[t]{2}{*}{ Assay 2} & EXP & Confluence & Standard medium for 1 week & $\begin{array}{l}\text { Osteogenic differentiation medium } \\
\text { with bFGF }\end{array}$ \\
\hline & CTR & Confluence & Standard medium for 1 week & Osteogenic differentiation medium \\
\hline \multirow[t]{3}{*}{ Assay 3} & EXP1 & Confluence & Standard medium with bFGF for 1 week & $\begin{array}{l}\text { Osteogenic differentiation medium } \\
\text { with bFGF }\end{array}$ \\
\hline & EXP2 & Confluence & Standard medium with bFGF for 1 week & Osteogenic differentiation medium \\
\hline & CTR & Confluence & Standard medium for 1 week & Osteogenic differentiation medium \\
\hline \multirow[t]{3}{*}{ Assay 4} & EXP1 & Confluence & Standard medium with bFGF for 2 weeks & $\begin{array}{l}\text { Osteogenic differentiation medium } \\
\text { with bFGF }\end{array}$ \\
\hline & EXP2 & Confluence & Standard medium with bFGF for 2 weeks & Osteogenic differentiation medium \\
\hline & CTR & Confluence & Standard medium for 2 weeks & Osteogenic differentiation medium \\
\hline \multirow[t]{2}{*}{ Assay 5} & EXP & Confluence & $\begin{array}{l}\text { Standard medium with bFGF for } 1 \text { week } \\
\text { and subcultivation }\end{array}$ & Osteogenic differentiation medium \\
\hline & CTR & Confluence & $\begin{array}{l}\text { Standard medium for } 1 \text { week and } \\
\text { subcultivation }\end{array}$ & Osteogenic differentiation medium \\
\hline
\end{tabular}

EXP, experimental; CTR, control; bFGF, basic fibroblastic growth factor. 
with bFGF (10 ng $\mathrm{mL}^{-1}$ ) for 2 weeks were designated as group 2, and the cells pre-treated with standard media without bFGF for 1 week were designated as the control group. After pre-treatment, the culture media in all of the groups were all changed to standard osteogenic differentiation medium. The ALP activity assay was performed on days 2, 4, 6, 8, 10, 12 and 14 after osteogenic differentiation, using an ALP assay kit (JianCheng Co., Nanjing, China) according to the manufacturer's instruction. The results were measured spectrophotometrically at $520 \mathrm{~nm}$

\section{Quantification Reverse Transcription Polymerase Chain Reaction (qRT-PCR)}

The DPSCs $\left(1 \times 10^{4}\right.$ cells per dish) were seeded onto $30 \mathrm{~mm}$ dishes and were also divided into group 1 , group 2 and the control group. After pre-treatment, the culture media in all of the groups were all changed to standard osteogenic differentiation medium. One week later, total RNA was extracted from the DPSCs using RNAiso Plus (Takala, Osaka, Japan) and was reverse transcribed using a Takala reverse transcriptase kit (Takala). Real-time PCR was performed using a standard SYBR Green PCR kit (Takala) and the Applied Biosystems 7500 Real-Time PCR System (ABI, Foster City, CA, USA), according to the respective manufacturers' instructions. $\beta$-actin was used as an internal control. The expressions of osteogenic differentiation marker mRNA, ALP, dentine sialophosphoprotein (DSPP), osteocalcin (OCN) and bone sialoprotein (BSP) were analyzed. Each sample was analyzed in triplicate. The $2^{-\Delta \Delta C t}$ method was used to quantify the relative levels of gene expression. The results represent the ratio between the expression in the control group and that in the bFGF pre-treatment groups $\left(\log _{10}\right.$ scale). The primer sequences are presented in Table 2.

\section{In vivo osteogenic differentiation assay}

Cell culture

The DPSCs $\left(1 \times 10^{6}\right.$ cells per dish) were seeded in 32 $10-\mathrm{cm}$ dishes and were divided into group 1 , group 2 , a control group and a group called group 3 , in which cells were pre-treated with bFGF $\left(10 \mathrm{ng} \mathrm{mL}^{-1}\right.$ ) for 1 week and were treated with standard medium for another week (Table 3). After pre-treatment, the cells in group 1, group 2 and the control group were
Table 2 Primer sequences

\begin{tabular}{ll}
\hline Primer & \multicolumn{1}{c}{ Sequence $\left(5^{\prime}-3^{\prime}\right)$} \\
\hline Actin-forward & TGGCACCCAGCACAATGAA \\
Actin-reverse & CTAAGTCATAGTCCGCCTAGAAGCA \\
ALP-forward & CCACGTCTTCACATTTGGT \\
ALP-reverse & AGACTGCGCCTGGTAGTTGT \\
DSPP-forward & TCACAAGGGAGAAGGGAATG \\
DSPP-reverse & TGCCATTTGCTGTCAGTTT \\
OCN-forward & GGCAGCGAGGTAGTGAAGAG \\
OCN-reverse & CTGGAGAGGAGCAGAACTGG \\
BSP-forward & AAAGTGAGAACGGGGAACCT \\
BSP-reverse & GATGCAAAGCCAGAATGGAT \\
\hline
\end{tabular}

DSPP, dentine sialophosphoprotein; OCN, osteocalcin; BSP, bone sialoprotein; ALP, alkaline phosphatase.

treated with standard osteogenic differentiation medium for 1 week.

Cell seeding and transplantation

After cultivation, the DPSCs $\left(1 \times 10^{7}\right.$ cells $)$ in each dish were seeded into HA/TCP (60/40) pills (diameter $=5 \mathrm{~mm}$, thickness $=1.5 \mathrm{~mm})$ and were transplanted subcutaneously into 16 naked mice, which had been averagely and randomly divided into four groups. Incision were made in the backs of each animal and then bilateral blunt dissection created two pouches. The pills were placed into each side of the back, which was then sutured. These procedures were performed in accordance with the specifications of an animal protocol approved by the Ethics Committee of the Fourth Military Medical University.

\section{Sectioning and staining}

The transplants were removed 2 and 3 months after transplantation, were fixed with $4 \%$ paraformaldehyde, and then cut into slices and stained using a Modified Ponceau Trichrome Stain (Oh et al. 2013). For histometric analysis of cartilage-like and bone-like tissue formation, computer-assisted histometric measurements were acquired with an automated image analysis, as previously described (Zhang et al. 2012).

\section{Statistical analysis}

The data are expressed as means \pm SDs. The statistical significance was evaluated using unpaired or paired Student's $t$-tests for comparisons between two means, whereas MANOVA was used to evaluate multiple means. A value of $P<0.05$ was assumed to denote statistical significance. 
Table 3 Culture method

\begin{tabular}{lccc}
\hline Group & Cell condition & Pre-culture & Osteogenic differentiation \\
\hline Negative control & Confluence & Standard medium for 1 week & Standard osteogenic differentiation \\
& & medium for 1 week & Standard medium for 1 week \\
Positive control & Confluence & Standard medium with bFGF for 1 week & Standard osteogenic differentiation \\
Group1 & Confluence & Standard medium with bFGF for 1 week & medium for 1 week \\
Group2 & Confluence & Standard medium with bFGF for 2 weeks & Standard osteogenic differentiation \\
& & & medium for 1 week
\end{tabular}

bFGF, basic fibroblastic growth factor.

\section{Results}

bFGF affected the osteogenic differentiation of DPSCs in a treatment-dependent manner

Basic fibroblastic growth factor affected the osteogenic differentiation of DPSCs in a treatment-dependent manner. In assay 1 , bFGF stimulation during the osteogenic differentiation period could upregulate the osteogenic differentiation ability of DPSCs (Fig. 1a). In assay 2, bFGF stimulation during the osteogenic differentiation period could upregulate the osteogenic differentiation ability of DPSCs, regardless of 1-week pre-treatment with standard medium (Fig. 1b). In assay 3, after 1 week of pre-treatment with bFGF, the osteogenic differentiation ability of DPSCs was increased, regardless of bFGF stimulation during the osteogenic differentiation period (Fig. 1c). In assay 4, after 2 weeks of pre-treatment with bFGF, the osteogenic differentiation ability of DPSCs was decreased, regardless of bFGF stimulation during the osteogenic differentiation period (Fig. 1d). In assay 5, after 1 week of pre-treatment with bFGF, the osteogenic differentiation ability of DPSCs was increased after subcultivation (Fig. 1e). According to the Alizarin red quantification results, the pre-treatment dura-
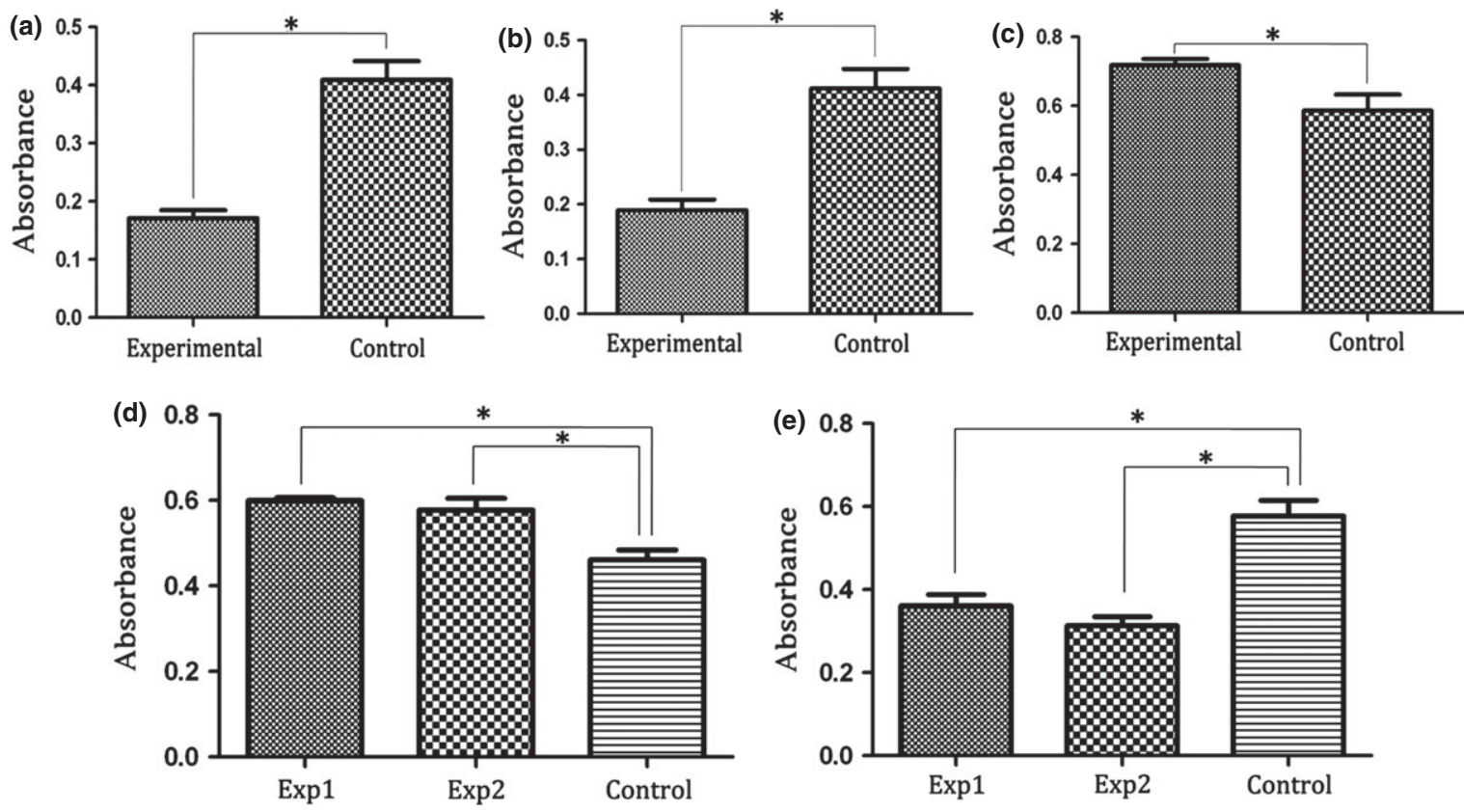

Figure 1 Alizarin red quantification assay results. Different basic fibroblastic growth factor (bFGF) treatment manners lead to different results of osteogenic differentiation of dental pulp stem cells (DPSCs) in alizarin red quantification assay 1 (a), 2 (b), 3 (c), 4 (d), 5 (e). The means and the standard errors of the means (SEMs) of three identical experiments are shown. *Indicates $P<0.05$ compared to the control group. 
tion was one of the key factors that determined how bFGF affected the osteogenic differentiation of the DPSCs. In assays 1, 2 and 4, bFGF inhibited the osteogenic differentiation of DPSCs. Assays 1 and 2 showed that bFGF stimulation in the osteogenic differentiation period decreased the in vitro osteogenic differentiation ability of DPSCs. In addition, no significant differences were found between cells that had just reached confluence and cells cultured for 1 week. There was no significant difference between cells that were not pre-treated with bFGF, and cells that were pre-treated with bFGF for 2 weeks in assay 4 . However, in assays 3 and 5, in which the DPSCs were pre-treated with bFGF for 1 week, the osteogenic differentiation of the DPSCs was enhanced. Assay 5 also showed that the impact of bFGF on DPSCs could be transferred to the next passage because assay 5 showed similar results to assay 3 after subculture.

Based on these results, ALP activity quantification and qRT-PCR were performed, which showed supplemental results. In both assays, group 1 refers to 1 week of bFGF pre-treatment, group 2 refers to 2 weeks of bFGF pre-treatment, and control group refers to 1 week of standard medium pre-treatment. ALP activity quantification was performed every 2 days from the initiation of osteogenic differentiation. Interestingly, the absorbance value (wavelength $=520 \mathrm{~nm}$ ) of group 1 was higher than that in the control group beginning on day 6 , whereas the absorbance value of group 2 was lower than that in the control group beginning on day 6 (Fig. 2a). qRT-PCR results showed that the expressions of ALP,
DSPP, OCN and BSP of the DPSCs in group 1, in which DPSCs were pre-treated with bFGF for 1 week, were higher than in the control group, and the expressions of ALP, DSPP, OCN and BSP of the DPSCs in group 2, in which DPSCs were pre-treated with bFGF for 2 week, were lower than in the control group (Fig. 2b).

bFGF affected the in vivo osteogenic differentiation of DPSCs depending on the duration of pre-treatment

Two months after transplantation

After 2 months, cartilage-like tissues (stained in blue) were formed in the edge of the hole in the control group (Fig. 3a), and there were cartilage-like tissues formed in the middle of the hole in group 1 (Fig. 3b). Obvious cartilage- or bone-like tissue formation was not observed in group 2 (Fig. 3c) or group 3 (Fig. 3d). However, well-developed vascular tissue was only observed in group 3. Histometric measurements showed that there were more cartilage-like tissues formed in group 1 than in the control group (Fig. 5a). In addition, little bone formation was observed in any of the groups (Fig. 5b).

Three months after transplantation

After 3 months, cartilage (stained in blue) - and bone-like (stained in red) tissue formation was observed in the control group (Fig. 4a) and in group 1(Fig. 4b). Obvious cartilage- or bone-like tissues
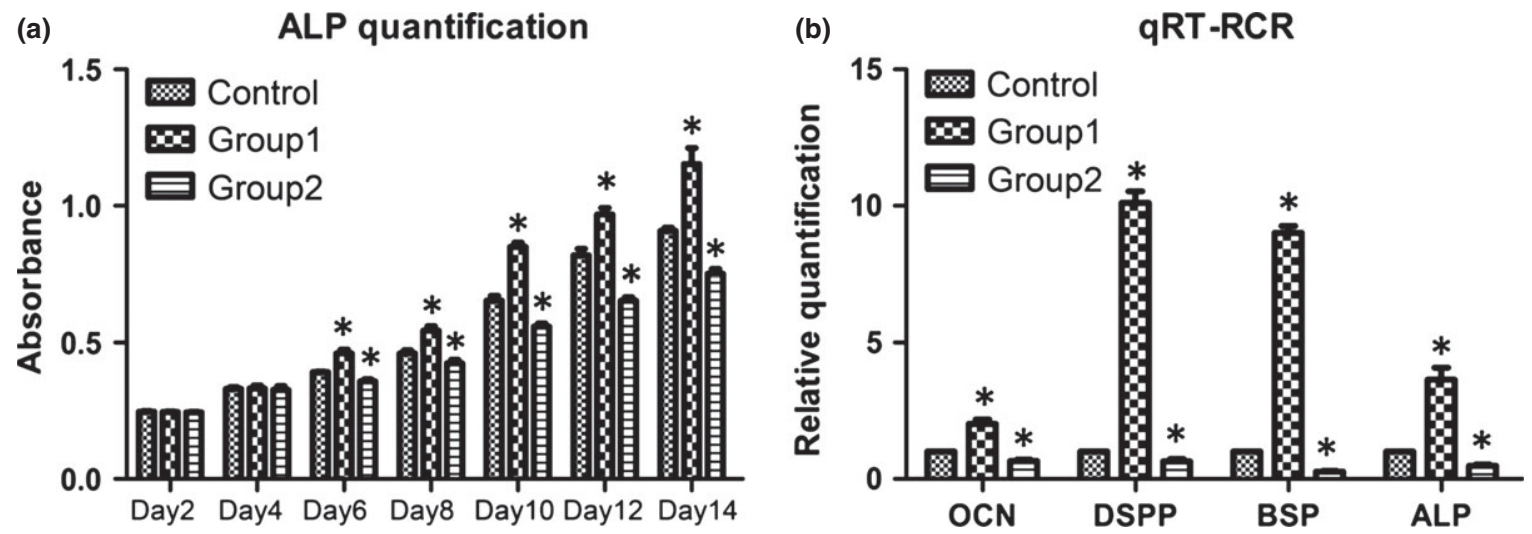

Figure 2 Alkaline phosphatase (ALP) activity quantification and qRT-PCR results. ALP activity quantification (a) and qRT-PCR (b) results showed that 1 week/2 weeks of pre-treatment with basic fibroblastic growth factor (bFGF) increased/decreased the osteogenic differentiation ability of dental pulp stem cells (DPSCs). The means and the standard errors of the means (SEMs) of three identical experiments are shown. *Indicates $P<0.05$ compared to the control group. 

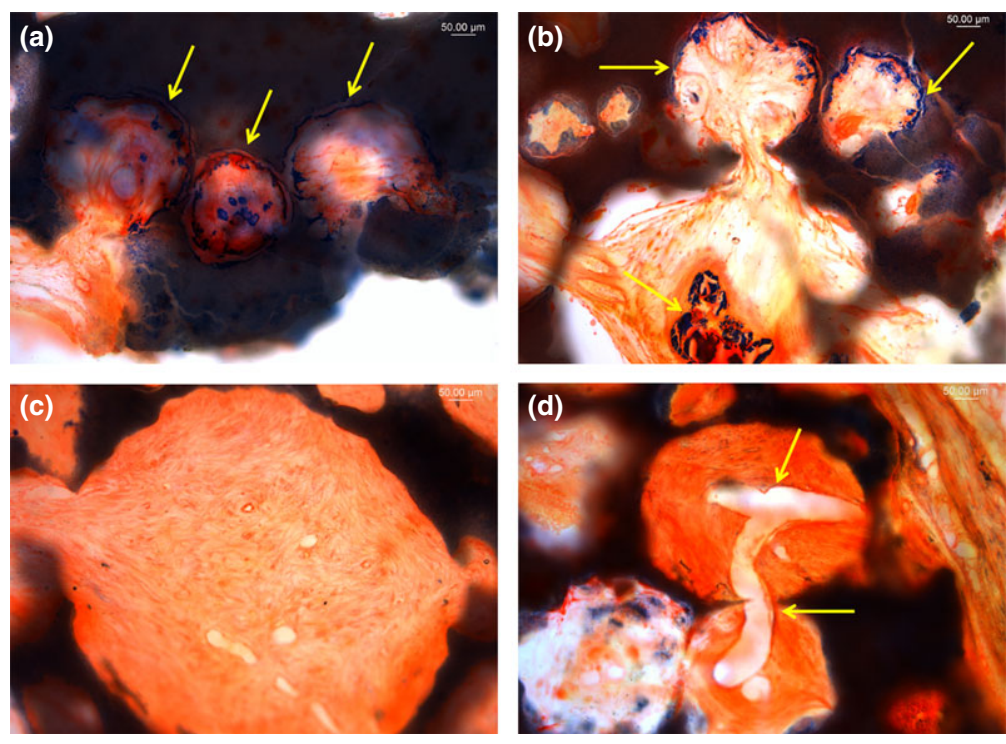

Figure 3 Two-month in vivo osteogenic differentiation assay. Different basic fibroblastic growth factor (bFGF) treatment manners lead to different results of osteogenic differentiation of dental pulp stem cells (DPSCs) in the in vivo osteogenic differentiation assay after 2 months transplantation.
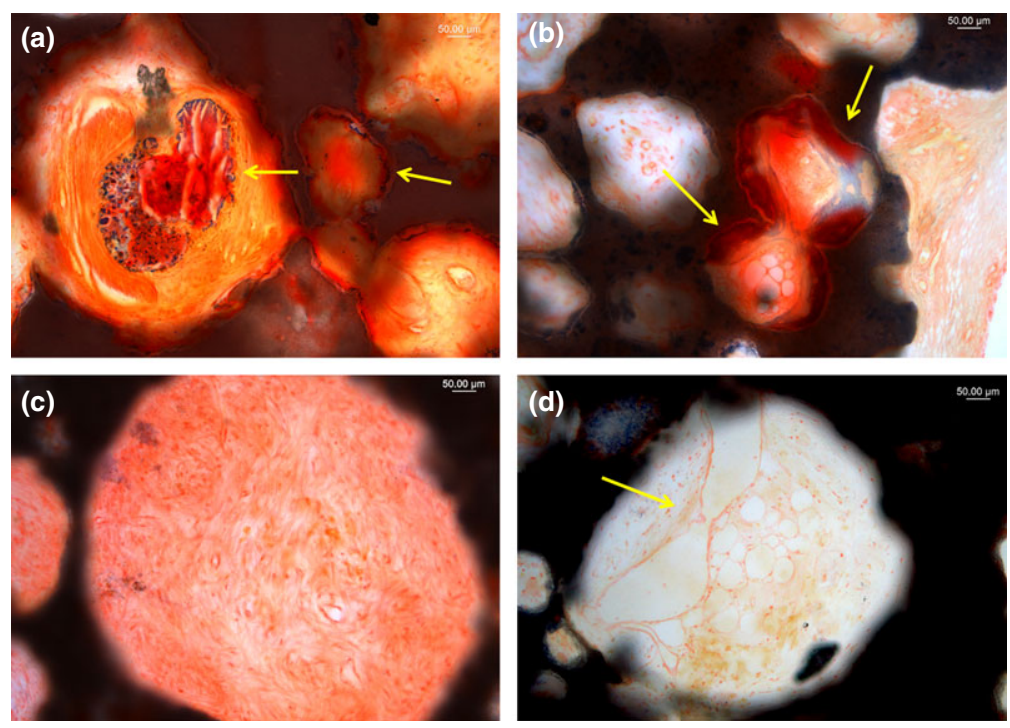

Figure 4 Three-month in vivo osteogenic differentiation assay. Different basic fibroblastic growth factor (bFGF) treatment manners lead to different results of osteogenic differentiation of dental pulp stem cells (DPSCs) in the in vivo osteogenic differentiation assay after 3 months transplantation.

formation was also not observed in group 2 (Fig. 4c) or group 3 (Fig. 4d). There was well-developed vascular tissue formation in group 3, which was not observed in the other groups. Histometric measurements showed that there were more bone-like tissues formed in group 1 than in the control group
(Fig. 5d), but there were no significant differences in the cartilage-like tissue formation between group 1 and the control group (Fig. 5c).

The in vivo results were similar to the in vitro results. Thus, this experiment confirmed the results of the in vitro experiments. 
(a) 2 monthes cartilage-like tissue formation

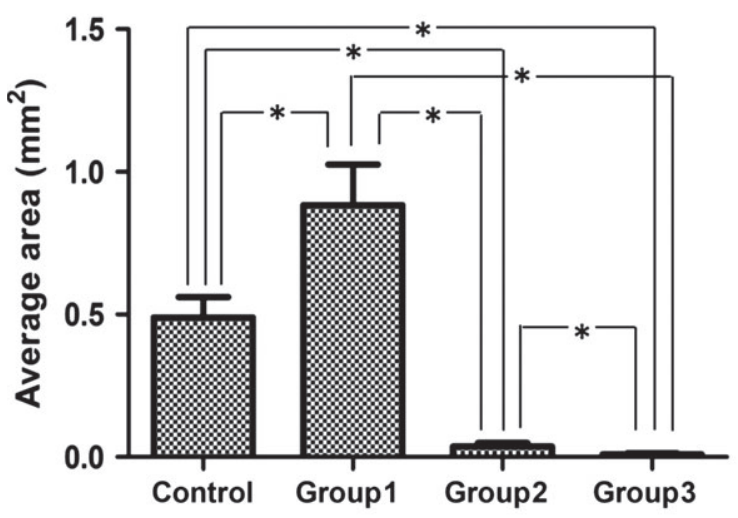

3 month cartilage-like tissue formation

(c) 1.57

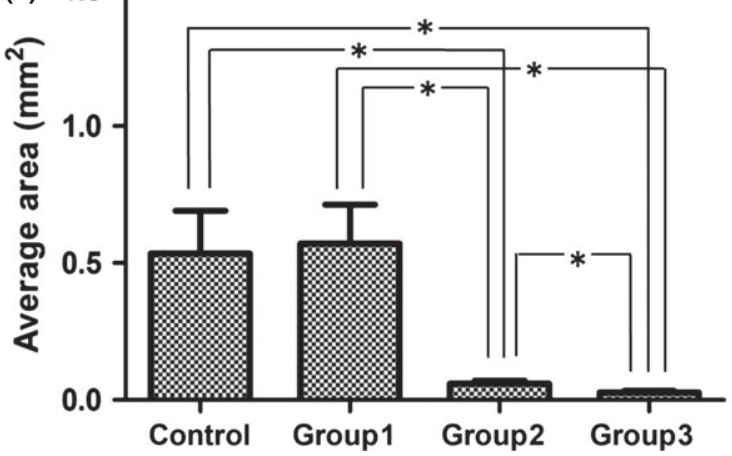

(b)

2 month bone-like tissue formation

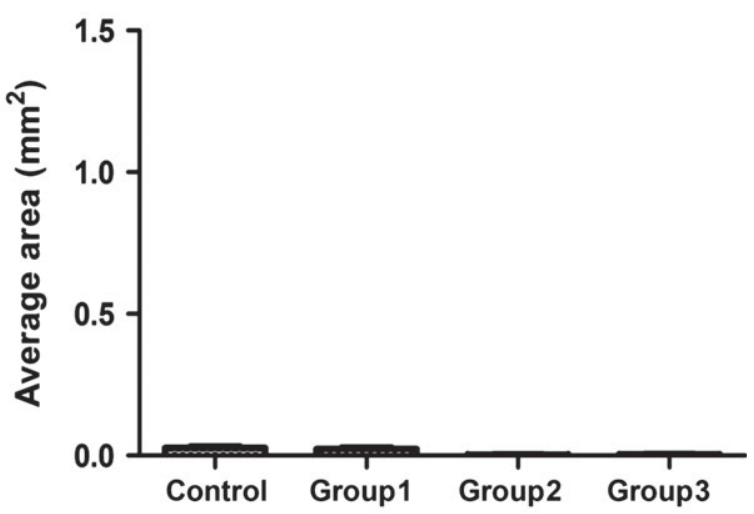

3 month bone-like tissue formation

(d)

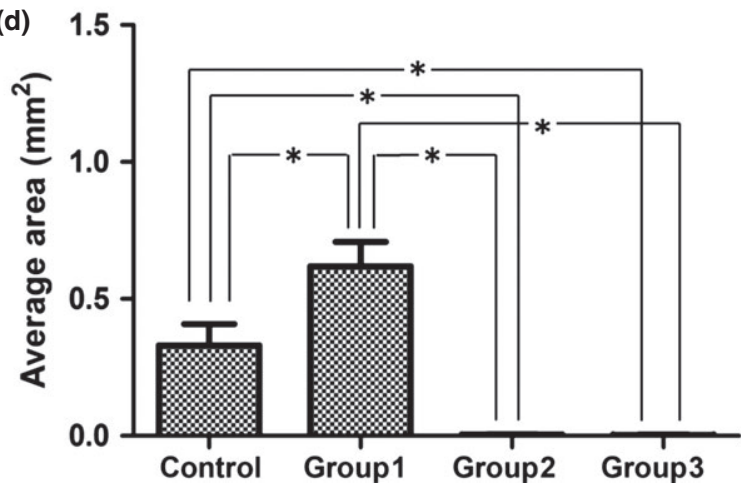

Figure 5 Quantitative analysis of the in vivo osteogenic differentiation assay. The results of the in vivo osteogenic differentiation assays were statistically confirmed by histometric measurements. *Indicates $P<0.05$.

\section{Discussion}

Several interesting and confusing results appear in the literature, which indicate that the nature of bFGF action is complex and that the biological effects of bFGF could depend on the differentiation stage of osteoblasts and the length and mode of exposure to certain factors (Fakhry et al. 2005). In the present study, an experiment was performed on how different bFGF stimulation conditions affected the osteogenic differentiation of DPSCs, and so, 1 and 2 weeks of stimulation of bFGF was chosen as the treatment protocol because these durations have been frequently used in previous studies (Shiba et al. 1995, He et al. 2008, Shimabukuro et al. 2009, Osathanon et al. 2011). The Alizarin red quantification assay was divided into five small assays. The main assay division indicated the differences between the stimulation time and order. The bFGF stimulation of DPSCs was divided into the pre-treatment period and the osteogenic differentiation period. As observed from the results, the difference in the stimulation time and order led to different osteogenic differentiation abilities of DPSCs. bFGF treatment did not stimulate proliferation of human calvarial bone cells that had been grown in mineralizing medium for several weeks (Debiais et al. 1998). As shown in assays 1 and 2, bFGF stimulation during the osteogenic differentiation period decreased the osteogenic differentiation of DPSCs, regardless of the cell condition (confluence or multilayer). Fakhry et al. (2005) found that the mitogenic effects of bFGF were more evident in maturing osteoblasts than in osteogenic pre-cursor cells. Assay 4 showed that 2 weeks of pre-treatment with bFGF decreased the osteogenic differentiation ability of DPSCs regardless of bFGF stimulation during the osteogenic differentiation period. However, the lack of mitogenic effect might have resulted from the onset of mineralization (Fakhry et al. 2005). With 1 week of pre-treatment with $\mathrm{bFGF}$, the osteogenic differentiation ability of the 
DPSCs was increased regardless of bFGF stimulation during osteogenic differentiation period. Assays 3 and 4 indicated that the pre-treatment period was vital for the osteogenic differentiation of DPSCs in vitro. Assay 5 showed that the impact of bFGF could be inherited. In other words, these phenomena indicated that the impact of bFGF on DPSCs occurred at the genetic level and was not abolished by subcultivation. According to these results, it can be concluded that 1 week of pretreatment with bFGF increased the in vitro osteogenic differentiation ability of DPSCs. However, these effects were not time dependent: 2 weeks of pre-treatment with bFGF decreased the in vitro osteogenic differentiation ability of DPSCs. This finding could have been due to bFGF playing different roles depending on the differentiation stage of DPSCs into osteoblasts (Fakhry et al. 2005). In addition, the ALP activity quantification assay and qRT-PCR results also confirmed that the pre-treatment period was vital for the osteogenic differentiation of DPSCs in vitro.

In the in vivo experiments, the same results were obtained. One week of pre-treatment with bFGF increased the formation of cartilage- and bone-like tissues, whereas 2 weeks of pre-treatment with bFGF decreased cartilage- and bone-like tissue formation. In the experiments, cell-scaffold complexes were collected after 2 and 3 months. After 2 months, the cartilage- and bone-like tissue formation of the DPSCs was not obvious, even in the control group and in group 1. After 3 months, cartilage- and bone-like tissues were remarkably formed in the control group and in group 1. However, group 2 and group 3 still exhibited no significant cartilage- or bone-like tissue formation. Therefore, it was concluded that obvious cartilage- and bone-like tissue formation could be observed after 3 months; thus, the in vivo experiment should last 3 months. It was also concluded that 1 week of pre-treatment with bFGF could increase the in vivo osteogenic differentiation ability of DPSCs, whereas 2 weeks of pretreatment with bFGF would decrease the in vivo osteogenic differentiation ability of DPSCs.

The wound healing process is activated when dentine injury occurs. The wound healing process could offer another explanation for the results. Previous research has shown that bFGF played an important role in the wound healing process (Smith 2002, Tran-Hung et al. 2008). The pre-treatment period in the present study could be considered the preliminary stage of wound healing. During this stage, there was little osteogenic differentiation induction component in the cellular matrix. The main function of bFGF was the promotion of the proliferation and migration of DPSCs (Shimabukuro et al. 2009). According to the results, 1 week of pre-treatment with bFGF could promote the osteogenic differentiation of DPSCs, and this result indicated that DPSCs could differentiate into osteoblast-like cells more easily with stimulation by bFGF and participate in the preliminary stages of wound healing. Previous research showed that 2 weeks of stimulation with bFGF could attenuate the osteogenic differentiation-related mRNA expression of collagen type I, osteonectin, BSP and DSPP (Shimabukuro et al. 2009). These results were similar to those of the present study. Furthermore, Osathanon et al. (2011) reported that 2 weeks of bFGF stimulation would enhance the stemness of DPSCs by upregulating OCT4, NANOG1 and REX1, which was an additional finding that indicated that 2 weeks of bFGF stimulation could attenuate the osteogenic differentiation of DPSCs. Thus, it can be deduced that bFGF can activate DPSCs by upregulating the proliferation, migration and osteogenic differentiation of DPSCs during the preliminary stage of wound healing. In contrast, bFGF attenuates the osteogenic differentiation and maintains the stemness of the DPSCs, which is not activated in the preliminary stages of wound healing. This mechanism will maintain the population of DPSCs in the dental pulp during the wound healing process.

The osteogenic differentiation of dental pulp tissue is a double-edged sword. When dental pulp is subjected to harmful stimulation, the osteogenic differentiation of the odontoblasts or the odontoblast-like cells can form reactive or reparative dentine, which protects the dental pulp from further damage. It is known that DPSCs can differentiate into odontoblasts or odontoblast-like cells. However, during age-related changes and chronic inflammation of the dental pulp, the osteogenic differentiation of dental pulp cells will produce pulp stones and will form calcifications in the dental pulp cavity and root canal, which will make root canal treatment more complicated. It is known that DPSCs are mostly located in multicellular layers, and that these cells are relevant to the calcification obtained after calcium hydroxide pulp capping and pulpotomy. Therefore, the present study found a new method to control the osteogenic differentiation of dental pulp tissue with bFGF, to obtain the desired osteogenic differentiation state, which could bring great changes to the field of endodontic tissue engineering and endodontic therapy. 


\section{Conclusion}

One week of pre-treatment with bFGF increased the in vitro and in vivo osteogenic differentiation ability of DPSCs, whereas 2 weeks of pre-treatment with bFGF decreased the in vitro and in vivo osteogenic differentiation ability of DPSCs. Furthermore, the pre-treatment duration was vital for the osteogenic differentiation of DPSCs in vitro and in vivo. bFGF affected the osteogenic differentiation of DPSCs in a treatment-dependent manner both in vitro and in vivo. The data suggest that different treatment periods and durations could have opposite effects when the same cells were treated with the same cytokines. For future studies of DPSCs and bFGF, the stature of the DPSCs and treatment protocol with bFGF must be the primary considerations for all of the variables because a slight change in the stature of the DPSCs or the treatment protocol with bFGF could result in great changes to the result when DPSCs were treated by bFGF.

\section{Acknowledgements}

This work was supported by grants from the National Natural Science Foundation of China (No. 81170946, 81371139) and National Key Technologies R\&D Programme of the twelve-five Year Plan, the Ministry of Science and Technology of China (2012BAI07B03).

\section{References}

Abraham JA, Whang JL, Tumolo A et al. (1986) Human basic fibroblast growth factor nucleotide sequence and genomic organization. The EMBO Journal 5, 2523-8.

Canalis E, Lorenzo J, Burgess WH, Maciag T (1987) Effects of endothelial cell growth factor on bone remodelling in vitro. The Journal of Clinical Investigation 79, 52-8.

Canalis E, Centrella M, McCarthy T (1988) Effects of basic fibroblast growth factor on bone formation in vitro. The Journal of Clinical Investigation 81, 1572-7.

Cuny R, Jeanny JC, Courtois Y (1986) Lens regeneration from cultured newt irises stimulated by retina-derived growth factors (EDGFs). Differentiation 32, 221-9.

Davidson JM, Klagsbrun M, Hill KE et al. (1985) Accelerated wound repair, cell proliferation, and collagen accumulation are produced by a cartilage-derived growth factor. The Journal of Cell Biology 100, 1219-27.

Debiais F, Hott M, Graulet AM, Marie PJ (1998) The effects of fibroblast growth factor- 2 on human neonatal calvaria osteoblastic cells are differentiation period specific. Journal of Bone and Mineral Research 13, 645-54.
Fakhry A, Ratisoontorn C, Vedhachalam C et al. (2005) Effects of FGF-2/-9 in calvarial bone cell cultures: differentiation stage-dependent mitogenic effect, inverse regulation of BMP-2 and noggin, and enhancement of osteogenic potential. Bone 36, 254-66.

Gronthos S, Mankani M, Brahim J, Robey PG, Shi S (2000) Postnatal human dental pulp stem cells (DPSCs) in vitro and in vivo. Proceedings of the National Academy of the Sciences of the United States of America 97, 13625-30.

Hanada K, Dennis JE, Caplan AI (1997) Stimulatory effects of basic fibroblast growth factor and bone morphogenetic protein-2 on osteogenic differentiation of rat bone marrow-derived mesenchymal stem cells. Journal of Bone and Mineral Research 12, 1606-14.

He H, Yu J, Liu Y et al. (2008) Effects of FGF2 and TGF $\beta_{1}$ on the differentiation of human dental pulp stem cells in vitro. Cell Biology International 32, 827-34.

Kim YS, Min KS, Jeong DH, Jang JH, Kim HW, Kim EC (2010) Effects of fibroblast growth factor-2 on the expression and regulation of chemokines in human dental pulp cells. Journal of Endodontics 36, 1824-30.

Lobb RR, Alderman EM, Fett JW (1985) Induction of angiogenesis by bovine brain derived class I heparin-binding growth factor. The Journal of Biochemistry 24, 4969-73.

Mayahara H, Ito T, Nagai H et al. (1993) In vivo stimulation of endosteal bone formation by basic fibroblast growth factor in rats. Growth Factors 9, 73-80.

Nakamura T, Hanada K, Tamura M et al. (1995) Stimulation of endosteal bone formation by systemic injections of recombinant basic fibroblast growth factor in rats. Endocrinology 13, 1276-84.

Oh SH, Kim JR, Kwon GB, Namgung U, Song KS, Lee JH (2013) Effect of surface pore structure of nerve guide conduit on peripheral nerve regeneration. Tissue Engineering Part C: Methods 3, 233-43.

Osathanon T, Nowwarote N, Pavasant P (2011) Basic fibroblast growth factor inhibits osteogenic differentiation but induces neuronal differentiation by human dental pulp stem cells through a FGFR and PLC $\gamma$ signaling pathway. Journal of Cellular Biochemistry 12, 1807-16.

Shi S, Bartold PM, Miura M, Seo BM, Robey PG, Gronthos S (2005) The efficacy of mesenchymal stem cells to regenerate and repair dental structures. Orthodontics $\mathcal{E}$ Craniofacal Research 8, 191-9.

Shiba H, Nakamura S, Shirakawa M et al. (1995) Effects of basic fibroblast growth factor on proliferation, the expression of osteonectin (SPARC) and alkaline phosphatase, and calcification in cultures of human pulp cells. Developmental Biology 170, 457-66.

Shimabukuro Y, Ueda M, Ozasa M et al. (2009) Fibroblast growth factor-2 regulates the cell function of human dental pulp cells. Journal of Endodontics 35, 1529-35.

Smith AJ (2002) Pulpal responses to caries and dental repair. Caries Research 36, 223-32. 
Tang KT, Capparelli C, Stein JL et al. (1996) Acidic fibroblast growth factor inhibits osteoblast differentiation in vitro: altered expression of collagenase, cell growth-related, and osteogenic differentiation-associated genes. Journal of Cellular Biochemistry 61, 152-66.

Tran-Hung L, Laurent P, Camps J, About I (2008) Quantification of angiogenic growth factors released by human dental cells after injury. Archives of Oral Biololy 53, 9-13.

Tsutsumi S, Shimazu A, Miyazaki K et al. (2001) Retention of multilineage differentiation potential of mesenchymal cells during proliferation in response to FGF. Biochemical and Biophysical Research Communications 288, 413-9.

Varghese S, Ramsby ML, Jeffrey JJ, Canalis E (1995) Basic fibroblast growth factor stimulates expression of interstitial collagenase and inhibitors of metalloproteinases in rat bone cells. Endocrinology 136, 2156-62.

Zellin G, Linde A (2000) Effects of recombinant human fibroblast growth factor-2 on osteogenic cell populations during orthopic osteogenesis in vivo. Bone 26, 161-8.

Zhang W, Walboomers XF, van Kuppevelt TH, Daamen WF, Bian Z, Jansen JA (2006) The performance of human dental pulp stem cells on different three-dimensional scaffold materials. Biometerials 27, 5658-68.

Zhang J, An Y, Gao LN, Zhang YJ, Jin Y, Chen FM (2012) The effect of aging on the pluripotential capacity and regenerative potential of human periodontal ligament stem cells. Biometerials 33, 6974-86. 